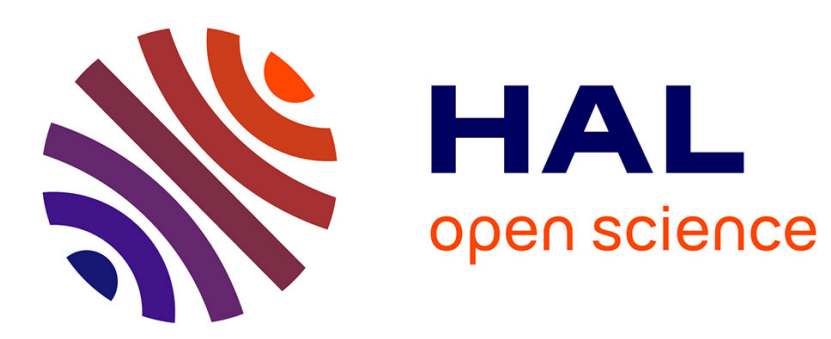

\title{
Generalized Iterated Fission Probability for Monte Carlo eigenvalue calculations
}

\author{
N. Terranova, A. Zoia
}

\section{To cite this version:}

N. Terranova, A. Zoia. Generalized Iterated Fission Probability for Monte Carlo eigenvalue calculations. Annals of Nuclear Energy, 2017, 108, pp.57-66. 10.1016/j.anucene.2017.04.014 cea-02421898

\section{HAL Id: cea-02421898 https://hal-cea.archives-ouvertes.fr/cea-02421898}

Submitted on 20 Dec 2019

HAL is a multi-disciplinary open access archive for the deposit and dissemination of scientific research documents, whether they are published or not. The documents may come from teaching and research institutions in France or abroad, or from public or private research centers.
L'archive ouverte pluridisciplinaire HAL, est destinée au dépôt et à la diffusion de documents scientifiques de niveau recherche, publiés ou non, émanant des établissements d'enseignement et de recherche français ou étrangers, des laboratoires publics ou privés. 


\title{
Generalized Iterated Fission Probability for Monte Carlo criticality simulations
}

\author{
Nicholas Terranova ${ }^{\mathrm{a}}$, Andrea Zoia ${ }^{\mathrm{a}, *}$ \\ ${ }^{a}$ Den-Service d'études des réacteurs et de mathématiques appliquées (SERMA), CEA, Université Paris-Saclay, F-91191, Gif-sur-Yvette, France
}

\begin{abstract}
The so-called Iterated Fission Probability (IFP) method has provided a major breakthrough for the calculation of the adjoint flux and more generally of adjoint-weighted scores in Monte Carlo criticality simulations. So far, IFP has been exclusively devoted to the analysis of the standard $k$-eigenvalue equation, by resorting to a formal identification between the adjoint fundamental eigenmode $\varphi_{k}^{\dagger}$ and the neutron importance $I_{k}$. In this work, we extend the IFP method to the $\alpha$-eigenvalue equation, enabling the calculation of the adjoint fundamental eigenmode $\varphi_{\alpha}^{\dagger}$ and the associated adjoint-weighted scores, including kinetics parameters. Such generalized IFP method is first verified in a simple two-group infinite medium transport problem, which admits analytical solutions. Then, $\alpha$-adjoint-weighted kinetics parameters are computed for a few reactor configurations by resorting to the Monte Carlo code TRIPOLI- $4{ }^{\circledR}$, and compared to the $k$-adjoint-weighted kinetics parameters obtained by the standard IFP. The algorithms that we have developed might be of interest in the interpretation of reactivity measurements, in the context of reactor period calculations by Monte Carlo simulation.
\end{abstract}

Keywords: IFP, Monte Carlo, $\alpha$ eigenvalues, kinetics parameters, adjoint, importance, TRIPOLI-4 ${ }^{\circledR}$

\section{Introduction}

In many technological applications, such as for instance start-up of commercial reactors (Pfeiffer et al., 1974), analysis of accelerator-driven systems (Persson et al., 2008), material control and accountability in critical assemblies (Sanchez and Jaegers, 1998), and pulsed neutron reactivity measurements (Cao and Lee, 2010), knowing the long-time evolution of neutron population is mandatory (Bell and Glasstone, 1970, Duderstadt and Martin, 1979). The integral-differential formulation of the time-dependent Boltzmann equation for the neutron flux $\varphi(\mathbf{r}, \mathbf{v}, t)$ can be written as follows (Bell and Glasstone, 1970)

$$
\begin{aligned}
\frac{1}{\mathrm{v}} \frac{\partial}{\partial t} \varphi(\mathbf{r}, \mathbf{v}, t)+L \varphi(\mathbf{r}, \mathbf{v}, t)= & F_{p} \varphi(\mathbf{r}, \mathbf{v}, t)+ \\
& \sum_{j} \chi_{d}^{j}(\mathbf{r}, v) \lambda_{j} c_{j}(\mathbf{r}, t)+\mathcal{S},
\end{aligned}
$$

coupled to the equations for precursor concentrations $c_{j}(\mathbf{r}, t)$

$$
\frac{\partial}{\partial t} c_{j}(\mathbf{r}, t)=\int v_{d}^{j}\left(\mathrm{v}^{\prime}\right) \Sigma_{f}\left(\mathbf{r}, \mathrm{v}^{\prime}\right) \varphi\left(\mathbf{r}, \mathbf{v}^{\prime}, t\right) \mathrm{d} \mathbf{v}^{\prime}-\lambda_{j} c_{j}(\mathbf{r}, t)
$$

where the net disappearance operator $L$ and the prompt fission operator $F_{p}$ are respectively defined as

$$
\begin{gathered}
L f=\mathbf{\Omega} \cdot \nabla f+\Sigma_{t} f-\int \Sigma_{s}\left(\mathbf{r}, \mathbf{v}^{\prime} \rightarrow \mathbf{v}\right) f\left(\mathbf{r}, \mathbf{v}^{\prime}\right) \mathrm{d} \mathbf{v}^{\prime}, \\
F_{p} f=\chi_{p}(\mathbf{r}, \mathrm{v}) \int v_{p}\left(\mathrm{v}^{\prime}\right) \Sigma_{f}\left(\mathbf{r}, \mathbf{v}^{\prime}\right) f\left(\mathbf{r}, \mathbf{v}^{\prime}\right) \mathrm{d} \mathbf{v}^{\prime}
\end{gathered}
$$

${ }^{*}$ Corresponding author. Tel. +33 (0)1 69089544

Email address: andrea.zoia@cea.fr (Andrea Zoia)
In the equations above, $\Sigma_{t}$ represents the total macroscopic cross section, $\Sigma_{s}\left(\mathbf{r}, \mathbf{v}^{\prime} \rightarrow \mathbf{v}\right)$ is the differential scattering crosssection, $\chi_{p}$ and $v_{p}$ are respectively the prompt fission neutron spectrum and the average multiplicity for a fissioning nucleus (in order to keep notation to a minimum, we are considering here a single fissile species: the generalization is straightforward). Analogously, $\chi_{d}^{j}$ and $v_{d}^{j}$ are the delayed neutron spectrum and multiplicity related to the precursor family $j$, while $\lambda_{j}$ is the associated decay constant. The problem is closed once proper initial and boundary conditions for $\varphi(\mathbf{r}, \mathbf{v}, t)$ and $c_{j}(\mathbf{r}, t)$ are assigned. All physical parameters (i.e., cross-sections and velocity spectra) have been here supposed time-independent.

Monte Carlo codes are considered the reference tools for esbe compared to faster, but approximated, deterministic calculations (Lux and Koblinger, 1991). Monte Carlo methods rely essentially on the simulation of random walks in position-velocity $\{\mathbf{r}, \mathbf{v}\}$ phase space through high fidelity descriptions of both geometry and physical laws for particle interaction with matter. In the last decades, the growing computer power has opened the possibility to solve the full time-dependent Boltzmann equation by Monte Carlo methods (Sjenitzer and Hoogenboom, 2013), providing the complete description of the neutron population evolving in a system, once initial conditions are provided.

Due to the computational cost of time-dependent simulations, especially when neutrons and precursors must be simultaneously taken into account, it is convenient to formally expand the time-dependent flux $\varphi(\mathbf{r}, \mathbf{v}, t)$ into a set of (supposedly complete 1 , orthogonal eigenfunctions depending only on $\mathbf{r}$ and $\mathbf{v}$, whose determination would in principle involve the

${ }^{1}$ Proving the feasibility of such an expansion (or even the simple existence 
simulation of time-independent equations. For instance, $k$ and $\alpha$-eigenfunctions have been proposed, leading to different ${ }_{45}$ formulations of the neutron transport problem (Cohen, 1958, Keepin, 1965). The so-called $k$-eigenmodes $\varphi_{k}$ associated to the Boltzmann equations have been widely used in the last 50 years (Goad and Johnston, 1959, Lux and Koblinger, 1991, Lewis and Miller, 1984): they stem from scaling the fission productions term by a factor $k$ and imposing exact criticality without external sources in Eq. (1) (i.e., the time derivatives for neutrons and precursors must vanish), which leads to

$$
L \varphi_{k}(\mathbf{r}, \mathbf{v})=\frac{1}{k} F_{p} \varphi_{k}(\mathbf{r}, \mathbf{v})+\frac{1}{k} F_{d} \varphi_{k}(\mathbf{r}, \mathbf{v})
$$

where we have introduced the delayed production operator

$$
F_{d}^{j} f=\chi_{d}^{j}(\mathbf{r}, \mathrm{v}) \int v_{d}^{j}\left(\mathrm{v}^{\prime}\right) \Sigma_{f}\left(\mathbf{r}, \mathrm{v}^{\prime}\right) f\left(\mathbf{r}, \mathbf{v}^{\prime}\right) \mathrm{d} \mathbf{v}^{\prime} .
$$

The terms $k$ represent the eigenvalues associated to $\varphi_{k}$. The socalled $\alpha$-eigenfunctions $\varphi_{\alpha}$ (Cohen, 1958, Bell and Glasstone, 1970; Duderstadt and Martin, 1979; Lewis and Miller, 1984) are introduced by requiring a separation of variables

$$
\varphi(\mathbf{r}, \mathbf{v}, t)=\sum_{\alpha} \varphi_{\alpha}(\mathbf{r}, \mathbf{v}) e^{\alpha t}, \quad c_{j}(\mathbf{r}, t)=\sum_{\alpha} c_{\alpha}^{j}(\mathbf{r}) e^{\alpha t},
$$

where $\alpha$ are the associated eigenvalues, and injecting these expressions into Eqs. (1) and (2). This leads to (Weinberg, 1952, Cohen, 1958, Henry, 1964, Bell and Glasstone, 1970)

$$
\begin{aligned}
& \frac{\alpha}{\mathrm{V}} \varphi_{\alpha}(\mathbf{r}, \mathbf{v})+L \varphi_{\alpha}(\mathbf{r}, \mathbf{v})= \\
& \quad=F_{p} \varphi_{\alpha}(\mathbf{r}, \mathbf{v})+\sum_{j} \frac{\lambda_{j}}{\lambda_{j}+\alpha} F_{d}^{j} \varphi_{\alpha}(\mathbf{r}, \mathbf{v}) .
\end{aligned}
$$

Eq. (8) may be actually more rigorously justified by applying Laplace transforms or equivalently performing spectral analy- ${ }^{50}$ sis (Duderstadt and Martin 1979).

In practice, one is often interested in determining the asympso totic behaviour of a system close to criticality, so that only the fundamental $k$-eigenmode $\varphi_{k_{0}}$ (with associated fundamental eigenvalue $k_{0}$ representing the ratio between neutron pop- ${ }^{55}$ ulation sizes at consecutive generations) or the fundamental $\alpha$ eigenmode $\varphi_{\alpha_{0}}$ (with associated fundamental eigenvalue $\alpha_{0}$ rep35 resenting the inverse of the asymptotic reactor period) are of interest, and faster-converging higher harmonics are typically neglected. Virtually all Monte Carlo transport codes for reactor calculations resort to power iteration in order to iteratively compute the fundamental eigen-pair $\left\{\varphi_{k_{0}}, k_{0}\right\}$ (Lux and Koblinger. 40 1991). The implementation of the modified power iteration algorithm leading to the fundamental eigen-pair $\left\{\varphi_{\alpha_{0}}, \alpha_{0}\right\}$ involves considerably higher computational costs (and convergence issues), and has thus received less attention so far (Cullen

of the fundamental mode) is not trivial in general. Precise, even if not very restrictive, conditions are required on the geometrical domain and on the material cross-sections (Bell and Glasstone ||1970||Duderstadt and Martin||1979||Larsen and Zweifel|1974). For the sake of simplicity, we assume that such conditions are met, which is typically the case for almost all systems of practical interest. et al., 2003; Brockway et al., 1985; Nauchi, 2014, Zoia et al. 2014, 2015).

Many key applications in reactor physics, including the calculation of kinetic parameters (Ussachoff, 1955, Keepin, 1965, Hetrick, 1971), reactivity coefficients and generalized sensitivities (Ussachoff, 1966, Gandini, 1978, 1981), additionally require the knowledge of the fundamental adjoint eigenmode. Extending the capabilities of Monte Carlo codes to adjointweighted scores has thus drawn the utmost attention in recent years. The rediscovery (Feghni et al., 2007, 2008, Nauchi and Kameyama, 2010, Kiedrowski, 2011b) of the so-called Iterated Fission Probability (IFP) interpretation of the adjoint flux, originally formulated by (Soodak, 1949, Weinberg, 1952, Ussachoff, 1955, Hurwitz, 1964), has actually enabled the exact calculation of the $\varphi_{k}^{\dagger}(\mathbf{r}, \mathbf{v})$ fundamental mode (Nauchi and Kameyama, 2010; Kiedrowski, 2011b; Shim et al., 2011), which satisfies

$$
L^{\dagger} \varphi_{k}^{\dagger}(\mathbf{r}, \mathbf{v})=\frac{1}{k} F_{p}^{\dagger} \varphi_{k}^{\dagger}(\mathbf{r}, \mathbf{v})+\frac{1}{k} F_{d}^{\dagger} \varphi_{k}^{\dagger}(\mathbf{r}, \mathbf{v}) .
$$

A number of production Monte Carlo codes, including MCNP (Kiedrowski, 2011a), SCALE (Perfetti, 2012), SERPENT (Leppanen, 2014) and TRIPOLI-4 ${ }^{\circledR}$ (Truchet et al., 2015), have integrated IFP algorithms.

In applications where an $\alpha$-eigenvalue formulation of the transport problem were to be deemed more appropriate, such as for instance reactor period calculations, the fundamental $\alpha$ adjoint flux $\varphi_{\alpha}^{\dagger}$ would be needed, satisfying

$$
\begin{aligned}
\frac{\alpha}{\mathrm{v}} \varphi_{\alpha}^{\dagger}(\mathbf{r}, \mathbf{v})+ & L^{\dagger} \varphi_{\alpha}^{\dagger}(\mathbf{r}, \mathbf{v})= \\
& =F_{p}^{\dagger} \varphi_{\alpha}^{\dagger}(\mathbf{r}, \mathbf{v})+\sum_{j} \frac{\lambda_{j}}{\lambda_{j}+\alpha}\left(F_{d}^{j}\right)^{\dagger} \varphi_{\alpha}^{\dagger}(\mathbf{r}, \mathbf{v})
\end{aligned}
$$

In this work we propose a generalized IFP method that can be used in order to estimate the adjoint fundamental flux $\varphi_{\alpha}^{\dagger}(\mathbf{r}, \mathbf{v})$ and more generally to compute adjoint-weighted functionals of the kind $\left\langle\varphi_{\alpha}^{\dagger}, \cdot\right\rangle$. The derivation of the algorithm is based on the connection between the power iteration, the adjoint equations, and the definition of the importance function.

This paper is organized as follows: in Sec. 2 we recall the basic structure of the modified $\alpha-k$ power iteration, and in Sec. 3 we illustrate the relation between the balance equation for the importance and the adjoint equation for the $\alpha$-eigenvalue expansion. Then, in Sec. 4 we show how these elements can be combined in order to provide a generalized IFP algorithm leading to the fundamental adjoint eigenmode $\varphi_{\alpha}^{\dagger}$. These results are first verified in Sec. 5 on a simple reactor model where exact reference solutions can be obtained, and then applied in Sec. 6 to the analysis of a few critical cores. Conclusions are finally drawn in Sec.7.

\section{Obtaining the fundamental direct eigen-pairs}

The fundamental eigen-pairs associated to Eq. (5) can be obtained by applying the standard Monte Carlo implementation of the power iteration algorithm (Lux and Koblinger, 1991). This 
basically amounts to assigning a guess distribution $S^{(0)}(\mathbf{r}, \mathbf{v})$ for the prompt and delayed fission source (the right hand side of Eq. (9)), and iteratively solving a fixed source problem

$$
L \varphi_{k}^{(g+1)}(\mathbf{r}, \mathbf{v})=S^{(g)}(\mathbf{r}, \mathbf{v})
$$

at each generation $g$. During transport, prompt and delayed neutrons emitted at fission events are recorded, and will pro-

70 vide the source for the next generation. The value $k^{(g)}$ is estimated as the ratio of the neutron population size at the end of each iteration to the source size, and nomalization is enforced by rescaling the initial population at generation $g+1$ by a factor ${ }_{100}$ $k^{(g)}$. This algorithm expresses a balance between the net disap75 pearance terms, collected at the left hand side of the equation, and production terms, collected at the right hand side. For large $g$, the eigen-pair estimate $\left\{\varphi_{k}^{(g)}, k^{(g)}\right\}$ converge to the fundamental eigen-pair $\left\{\varphi_{k_{0}}, k_{0}\right\}$.

The fundamental eigen-pair of Eq. (8) can be determined by 80 applying the so-called $\alpha-k$ power iteration (Brockway et al. 1985). Actually, it has been shown that the standard implementation of this algorithm in Monte Carlo codes may become unstable for negative dominant $\alpha_{0}$ and lead to anomalous termination (Nolen et al., 2012; Yamamoto and Miyoshi, 2003, Ye et al., 2006). The same problem has been reported also for deterministic solvers (Hill, 1983).

A remedy has been recently proposed in (Zoia et al., 2014 2015), by building upon an operator shift suggested in (Ye et al., 2006). The idea is to rearrange the terms in Eq. (8) so that it can be always interpreted as a balance equation between net disappearance on the left hand side and productions on the right hand side. This requires two alternative strategies, depending on the sign of the dominant $\alpha_{0}$. When $\alpha_{0}>0$, Eq. (8) can be formally rewritten as

$$
L_{\alpha} \varphi_{\alpha}=\frac{1}{k}\left[F_{p} \varphi_{\alpha}+\sum_{j} \frac{\lambda_{j}}{\lambda_{j}+\alpha} F_{d}^{j} \varphi_{\alpha}\right],
$$

where $L_{\alpha}=L+\Sigma_{\alpha}$ is a modified net disappearance operator, with an additional sterile capture cross section $\Sigma_{\alpha}=\alpha / \mathrm{v}>0$. The eigenvalue $\alpha$ has to be determined such that the fictitious parameter $k=k(\alpha)=1$. Then, the standard power iteration algorithm described above can be applied by starting from an initial tentative neutron distribution $S^{(0)}$ and providing a guess value $\alpha^{(0)}$ for $\alpha$. The corresponding $k$ eigenvalue is iteratively determined, and clearly depends on the current value of $\alpha$. The value of $\alpha$ for the next generation is adjusted until $k(\alpha)$ converges to $k=1$ : the corresponding value of $\alpha$ will provide the fundamental eigenvalue $\alpha_{0}$, associated to the fundamental eigenmode $\varphi_{\alpha_{0}}$ (Zoia et al. 2015).

When $\alpha_{0}<0, \Sigma_{\alpha}$ may become negative, and cannot be interpreted as a sterile capture cross-section. Eq. (8) is thus rearranged by preserving the balance between destructions and productions as (Zoia et al. 2015)

$$
L_{\alpha, \eta} \varphi_{\alpha}=\frac{1}{k}\left[F_{p} \varphi_{\alpha}+\sum_{j} \frac{\lambda_{j}}{\lambda_{j}+\alpha} F_{d}^{j} \varphi_{\alpha}+F_{\alpha, \eta} \varphi_{\alpha}\right],
$$

where we have formally introduced the modified net destruction operator $L_{\alpha, \eta}=L+\Sigma_{\alpha, \eta}$, with the $\alpha$-production cross section $\Sigma_{\alpha, \eta}=-\eta \alpha / \mathrm{v}>0, \eta$ being an arbitrary positive constant, and the associated $\alpha$-production operator $F_{\alpha, \eta}$

$$
F_{\alpha, \eta} f=\int v_{\eta} \delta\left(\mathbf{v}-\mathbf{v}^{\prime}\right) \Sigma_{\alpha, \eta} f\left(\mathbf{r}, \mathbf{v}^{\prime}\right) \mathrm{d} \mathbf{v}^{\prime},
$$

with

$$
v_{\eta}=\frac{\eta+1}{\eta}>0
$$

The term $v_{\eta}$ can be interpreted as the average number of (copy) neutrons produced by the $\alpha$-production operator having a delta kernel. The value $\alpha$ is updated as before (Zoia et al., 2015), and the whole procedure will eventually converge to the fundamental $\alpha$ eigen-pair, with the dominant eigenvalue lying in the interval $-\min \left[\lambda_{j}\right]<\alpha_{0}<0$.

When the system is exactly critical, we have $\alpha_{0}=0$ and $k_{0}=$ 1 , and the fundamental eigenmodes for the $\alpha$ - or $k$-eigenmode expansion coincide, i.e., $\varphi_{\alpha_{0}}=\varphi_{k_{0}}$.

\section{Relation between importance and adjoint equations}

In a multiplying system, the neutron importance $I(\mathbf{r}, \mathbf{v})$ is defined as the average number of descendant neutrons produced asymptotically in a distant generation by a single neutron initially injected at the phase space coordinates $\{\mathbf{r}, \mathbf{v}\}$ (Ussachoff. 1955: Henry, 1975). The neutron importance satisfies a balance equation (Ussachoff, 1955, Nauchi and Kameyama, 2010)

$$
I(\mathbf{r}, \mathbf{v})=p_{\mathrm{nc}} I(\mathbf{r}+\mathrm{d} \mathbf{s}, \mathbf{v})+Q(\mathbf{r}+\mathrm{d} \mathbf{s}, \mathbf{v}),
$$

where $p_{\mathrm{nc}}$ is the non-collision probability, and

$$
Q(\mathbf{r}, \mathbf{v})=\int \mathrm{d} \mathbf{v}^{\prime} q\left(\mathbf{r}, \mathbf{v} \rightarrow \mathbf{v}^{\prime}\right) I\left(\mathbf{r}, \mathbf{v}^{\prime}\right)
$$

is the average number of descendants for neutrons having a collision in $\mathrm{d} \mathbf{s}$, formally expressed in terms of a density $q$. We have denoted the infinitesimal path $\mathrm{d} \mathbf{s}=\mathbf{r}-\mathbf{r}^{\prime}$. The terms $p_{\mathrm{nc}}$ and $Q$ depend on the eigenfunction expansion that has been chosen to represent the neutron behaviour close to the critical state.

For $k$-eigenfunction expansion, we have $p_{\mathrm{nc}, \mathrm{k}}=1-\Sigma_{\mathrm{t}} \mathrm{d} s$. Moreover, neutrons are promoted to the next generation only by fission events, which yields

$$
\begin{gathered}
q_{k}\left(\mathbf{r}, \mathbf{v} \rightarrow \mathbf{v}^{\prime}\right)=\Sigma_{\mathrm{s}}\left(\mathbf{r}+\mathrm{d} \mathbf{s}, \mathbf{v} \rightarrow \mathbf{v}^{\prime}\right) \mathrm{d} s \\
+\frac{1}{k} \frac{\chi_{\mathrm{t}}\left(\mathrm{v} \rightarrow \mathrm{v}^{\prime}\right)}{4 \pi} v_{\mathrm{t}} \Sigma_{\mathrm{f}}(\mathbf{r}+\mathrm{d} \mathbf{s}, \mathrm{v}) \mathrm{d} s,
\end{gathered}
$$

where the fission term has been divided by $k$, since the importance of neutrons promoted at the next generation is rescaled precisely by this factor (Nauchi and Kameyama, 2010). Here $\chi_{\mathrm{t}}$ is the average total (prompt plus delayed) fission spectrum, and $v_{\mathrm{t}}$ is the total fission yield. Now, from Eqs. (17) and (16), by developing the total derivative along $\mathrm{d} \mathbf{s}$ we obtain

$$
\begin{aligned}
0 & =\Omega \cdot \nabla I_{k}(\mathbf{r}, \mathbf{v})-\Sigma_{\mathrm{t}} I_{k}(\mathbf{r}, \mathbf{v})+\int \mathrm{d} \mathbf{v}^{\prime} \Sigma_{\mathrm{s}}\left(\mathbf{r}, \mathbf{v} \rightarrow \mathbf{v}^{\prime}\right) I_{k}\left(\mathbf{r}, \mathbf{v}^{\prime}\right) \\
& +\frac{v_{\mathrm{t}} \Sigma_{\mathrm{f}}(\mathbf{r}, \mathrm{v})}{4 \pi k} \int \mathrm{d} \mathbf{v}^{\prime} \chi_{\mathrm{t}}\left(\mathrm{v} \rightarrow \mathrm{v}^{\prime}\right) I_{k}\left(\mathbf{r}, \mathbf{v}^{\prime}\right),
\end{aligned}
$$


where the index $k$ has been added to stress that this equation is valid when expanding in $k$-eigenfunctions. After a sufficiently large number of generations, $k \rightarrow k_{0}$. Then, by inspection, the neutron importance $I_{k}(\mathbf{r}, \mathbf{v})$ in Eq. (19) becomes proportional to the adjoint flux $\varphi_{k}^{\dagger}(\mathbf{r}, \mathbf{v})$, solution to the $k$-eigenvalue adjoint equation (9).

The same methodology can be applied to the $\alpha$ eigenfunctions. When $\alpha_{0}>0$, the term $\Sigma_{\alpha}$ must be added to the non-collision probability, i.e., $p_{\mathrm{nc}, \alpha}=1-\left(\Sigma_{\mathrm{t}}+\Sigma_{\alpha}\right) \mathrm{d} s$. Neutrons are promoted to the next generation only by fission, but delayed productions are modified by a factor depending on the $\alpha$ eigenvalue, as shown above. This leads to a modified source density

$$
\begin{aligned}
& q_{\alpha}\left(\mathbf{r}, \mathbf{v} \rightarrow \mathbf{v}^{\prime}\right)=\Sigma_{\mathrm{s}}\left(\mathbf{r}+\mathrm{d} \mathbf{s}, \mathbf{v} \rightarrow \mathbf{v}^{\prime}\right) \mathrm{d} s \\
& \quad+\frac{\chi_{\mathrm{p}}\left(\mathrm{v} \rightarrow \mathrm{v}^{\prime}\right)}{4 \pi k} v_{\mathrm{p}} \Sigma_{\mathrm{f}}(\mathbf{r}+\mathrm{d} \mathbf{s}, \mathrm{v}) \mathrm{d} s \\
& \quad+\sum_{j} \frac{\lambda_{j}}{\lambda_{j}+\alpha} \frac{\chi_{\mathrm{d}}^{j}\left(\mathrm{v} \rightarrow \mathrm{v}^{\prime}\right)}{4 \pi k} v_{\mathrm{d}}^{j} \Sigma_{\mathrm{f}}(\mathbf{r}+\mathrm{d} \mathbf{s}, \mathrm{v}) \mathrm{d} s,
\end{aligned}
$$

where $k$ is the ratio between two successive population sizes. Following the same procedure as above, the importance balance equation yields then

$$
\begin{aligned}
0 & =\Omega \cdot \nabla I_{\alpha}(\mathbf{r}, \mathbf{v})-\left(\Sigma_{\mathrm{t}}+\Sigma_{\alpha}\right) I_{\alpha}(\mathbf{r}, \mathbf{v}) \\
& +\int \mathrm{d} \mathbf{v}^{\prime} \Sigma_{\mathrm{s}}\left(\mathbf{r}, \mathbf{v} \rightarrow \mathbf{v}^{\prime}\right) I_{\alpha}\left(\mathbf{r}, \mathbf{v}^{\prime}\right) \\
& +\frac{v_{\mathrm{p}} \Sigma_{\mathrm{f}}(\mathbf{r}, \mathrm{v})}{4 \pi k} \int \mathrm{d} \mathbf{v}^{\prime} \chi_{\mathrm{p}}\left(\mathrm{v} \rightarrow \mathrm{v}^{\prime}\right) I_{\alpha}\left(\mathbf{r}, \mathbf{v}^{\prime}\right) \\
& +\sum_{j} \frac{\lambda_{j}}{\lambda_{j}+\alpha} \frac{v_{\mathrm{d}}^{j} \Sigma_{\mathrm{f}}(\mathbf{r}, \mathrm{v})}{4 \pi k} \int \mathrm{d} \mathbf{v}^{\prime} \chi_{\mathrm{d}}^{j}\left(\mathrm{v} \rightarrow \mathrm{v}^{\prime}\right) I_{\alpha}\left(\mathbf{r}, \mathbf{v}^{\prime}\right) .
\end{aligned}
$$

After a sufficiently large number of generations, $k \rightarrow 1$. This shows that for $\alpha_{0}>0$ the importance $I_{\alpha}$ solves the adjoint equation (12).

For $\alpha_{0}<0$, we will similarly define $p_{\mathrm{nc}, \alpha, \eta}=1-\left(\Sigma_{t}+\Sigma_{\alpha, \eta}\right) \mathrm{d} s$, and the source density will read

$$
\begin{aligned}
& q_{\alpha, \eta}\left(\mathbf{r}, \mathbf{v} \rightarrow \mathbf{v}^{\prime}\right)=\Sigma_{\mathrm{s}}\left(\mathbf{r}+\mathrm{d} \mathbf{s}, \mathbf{v} \rightarrow \mathbf{v}^{\prime}\right) \mathrm{d} s \\
& +\frac{\chi_{\mathrm{p}}\left(\mathrm{v} \rightarrow \mathrm{v}^{\prime}\right)}{4 \pi} v_{\mathrm{p}} \Sigma_{\mathrm{f}}(\mathbf{r}+\mathrm{d} \mathbf{s}, \mathrm{v}) \mathrm{d} s \\
& \quad+\sum_{j} \frac{\lambda_{j}}{\lambda_{j}+\alpha} \frac{\chi_{\mathrm{d}}^{j}\left(\mathrm{v} \rightarrow \mathrm{v}^{\prime}\right)}{4 \pi k} v_{\mathrm{d}}^{j} \Sigma_{\mathrm{f}}(\mathbf{r}+\mathrm{d} \mathbf{s}, \mathrm{v}) \mathrm{d} s \\
& \quad+\frac{v_{\eta}}{k} \delta\left(\mathbf{v}-\mathbf{v}^{\prime}\right) \Sigma_{\alpha, \eta}(\mathrm{v}) \mathrm{d} s .
\end{aligned}
$$

This finally yields the balance equation

$$
\begin{aligned}
0 & =\Omega \cdot \nabla I_{\alpha}(\mathbf{r}, \mathbf{v})-\left(\Sigma_{\mathrm{t}}+\Sigma_{\alpha, \eta}\right) I_{\alpha}(\mathbf{r}, \mathbf{v}) \\
& +\int \mathrm{d} \mathbf{v}^{\prime} \Sigma_{\mathrm{s}}\left(\mathbf{r}, \mathbf{v} \rightarrow \mathbf{v}^{\prime}\right) I_{\alpha}\left(\mathbf{r}, \mathbf{v}^{\prime}\right) \\
& +\frac{\nu_{\mathrm{p}} \Sigma_{\mathrm{f}}(\mathbf{r}, \mathrm{v})}{4 \pi k} \int \mathrm{d} \mathbf{v}^{\prime} \chi_{\mathrm{p}}\left(\mathrm{v} \rightarrow \mathrm{v}^{\prime}\right) I_{\alpha}\left(\mathbf{r}, \mathbf{v}^{\prime}\right) \\
& +\sum_{j} \frac{\lambda_{j}}{\lambda_{j}+\alpha} \frac{v_{\mathrm{d}}^{j} \Sigma_{\mathrm{f}}(\mathbf{r}, \mathrm{v})}{4 \pi k} \int \mathrm{d} \mathbf{v}^{\prime} \chi_{\mathrm{d}}^{j}\left(\mathrm{v} \rightarrow \mathrm{v}^{\prime}\right) I_{\alpha}\left(\mathbf{r}, \mathbf{v}^{\prime}\right) \\
& +\frac{v_{\eta}}{k} \Sigma_{\alpha, \eta}(\mathrm{v}) I_{\alpha}(\mathbf{r}, \mathbf{v}) .
\end{aligned}
$$

After a sufficiently large number of generations, $k \rightarrow 1$, which shows that for $\alpha_{0}<0$ the importance $I_{\alpha}$ solves the adjoint equation (13).

For an exactly critical multiplying system with $\alpha_{0}=0$, the equation for $I_{\alpha}$ is the same as for $I_{k}$ with $k_{0}=1$.

\section{A generalized IFP algorithm}

The formal identification between the neutron importance and the adjoint fundamental eigenmode has been the turning point in the development of the so-called Iterated Fission Probability (IFP) method (Feghni et al., 2007, 2008, Nauchi and Kameyama, 2010; Kiedrowski, 2011b). To the best of our knowledge, IFP has been exclusively applied to the $k$ equation: the idea is basically that the fundamental adjoint neutron flux $\varphi_{k}^{\dagger}\left(\mathbf{r}_{0}, \mathbf{v}_{0}\right)$ can be measured by injecting into a multiplying system a neutron at coordinates $\mathbf{r}_{0}, \mathbf{v}_{0}$ and recording the number of its descendants after $M$ generations, with $M \gg 1$ (Nauchi and Kameyama, 2010). This algorithm lations and thus does not require the simulation of backward random walks, which would be a daunting task for continuousenergy Monte Carlo methods (Hoogenboom, 2003). Since neutrons are promoted to the next generations only by fissions, the importance is a measure of the growth of the fission chains over $M$ latent generations (up to a rescaling factor $1 / k$ that is applied for population control). The span $M+1$ identifies the IFP cycle length (Nauchi and Kameyama, 2010, Kiedrowski, 2011b).

Before discussing how the IFP method can be extended to the $\alpha$-eigenvalue equation to determine the fundamental adjoint neutron flux $\varphi_{\alpha}^{\dagger}\left(\mathbf{r}_{0}, \mathbf{v}_{0}\right)$, let us first briefly recall the principles of this method as applied to the $k$-eigenvalue equation.

\subsection{Standard IFP for k-eigenvalue equations}

In the practical Monte Carlo implementation of the IFP algorithm, $I_{k}$ is estimated by running a fixed-source simulation over $M+1$ fission generations, for a single neutron starting at $\mathbf{r}_{0}, \mathbf{v}_{0}$. If $M$ is sufficiently large, the neutron population $(\pi)_{i}$ related to each ancestor $i$ reaches an asymptotic distribution, and the importance $I_{k}$ at generation $M+1$ can be thus obtained by collecting the simulation weights of all fission neutrons descending from their common ancestors. To prevent the neutron population from exploding or to go to extinction over the $M$ 
latent generations, a rescaling factor equal to $1 / k^{(g)}$ (the multiplication factor estimated at the latent generation $g$ ) is applied. The quantity $k^{(g)}$ asymptotically converges to the fundamental ${ }_{18}$ $k$-eigenvalue for large $M$, and the associated importance yields the fundamental adjoint neutron flux $\varphi_{k}^{\dagger}\left(\mathbf{r}_{0}, \mathbf{v}_{0}\right)$, up to a normalization factor. Adjoint-weighted sources $\left\langle\varphi_{k}^{\dagger}, \mathcal{S}\right\rangle$ can be similarly computed by convoluting the results for a single neutron with arbitrary initial distributions $\mathcal{S}$.

More generally, one is often led to consider bi-linear forms ${ }^{190}$ of the kind $\left\langle\varphi_{k}^{\dagger}, A \varphi_{k}\right\rangle$, involving both the fundamental $k$ eigenmode and the associated adjoint fundamental eigenmode, for a given operator $A$. An important application is the calculation of adjoint-weighted kinetic parameters (Kiedrowski, 2011a; Leppanen, 2014, Truchet et al., 2015), which are crucial ${ }^{19}$ for reactor operation and safety assessment (Bell and Glasstone, 1970, Keepin, 1965). When using the $k$-eigenmodes expansion, the effective generation time and the effective delayed neutron fraction per family $j$ are defined as

$$
\Lambda_{\text {eff }}=\frac{\left\langle\varphi_{k}^{\dagger}, \frac{1}{\mathrm{v}} \varphi_{k}\right\rangle}{\left\langle\varphi_{k}^{\dagger}, F \varphi_{k}\right\rangle}, \quad \beta_{\mathrm{eff}, \mathrm{j}}=\frac{\left\langle\varphi_{k}^{\dagger}, F_{\mathrm{d}}^{j} \varphi_{k}\right\rangle}{\left\langle\varphi_{k}^{\dagger}, F \varphi_{k}\right\rangle}
$$

respectively, with $\beta_{\mathrm{eff}}=\sum_{j} \beta_{\mathrm{eff}, \mathrm{j}}$. We have introduced the total fission operator $F=F_{\mathrm{p}}+F_{\mathrm{d}}$.

The IFP method is then integrated in a standard Monte Carlo power iteration: once the fundamental eigenmode $\varphi_{k}$ is attained, at each successive generation $g$ a neutron is labelled as an ancestor. The corresponding importance $I_{k}$ of this ancestor is estimated at a later generation $g+M+1$ via the IFP method.210 Then, the scalar products involved in Eq. 24) are computed by multiplying the score associated to the ancestor (pertaining to generation $g$ ) by its importance. The adjoint-weighted total and delayed fission terms may be then calculated as follows (see, e.g., Ref. (Truchet et al., 2015) for further details)

$$
\begin{gathered}
\left\langle\varphi_{k}^{\dagger}, F \varphi_{k}\right\rangle \propto k \sum_{i}(\pi)_{i}, \\
\left\langle\varphi_{k}^{\dagger}, F_{\mathrm{d}}^{j} \varphi_{k}\right\rangle \propto k \sum_{i}\left(\pi_{\mathrm{d}, \mathrm{j}}\right)_{i},
\end{gathered}
$$

where $\left(\pi_{\mathrm{d}, \mathrm{j}}\right)_{i}$ denotes the importance contribution due to ancestor delayed neutrons pertaining to family $j$ alone, and the summation is intended over the whole ancestor population. Similarly, the adjoint-weighted total neutron lifetime can be estimated as

$$
\left\langle\varphi_{k}^{\dagger}, \frac{1}{\mathrm{~V}} \varphi_{k}\right\rangle \propto \sum_{i}(\pi)_{i} t_{i}
$$

where $t_{i}$ is the lifetime of the ancestor neutron yielding the importance $(\pi)_{i}$, scored from its birth during generation $g$.

175 The IFP method for the $k$-eigenvalue equation recalled above has been practically integrated in production Monte Carlo codes for several purposes. General adjoint-weighted scores (Nauchi and Kameyama, 2010, Kiedrowski, 2011b; Shim et al., 2011, Kiedrowski, 2011a; Perfetti. 2012; Leppanen, 2014; Truchet

180 et al. 2015), including sensitivity profiles based on firstorder perturbations (Kiedrowski, 2011b) and exact perturbations (Truchet, 2014a b) can be currently estimated through IFP calculations. An overlapping-cycles algorithm has been proposed in the Monte Carlo codes SERPENT (Leppanen, 2014) and TRIPOLI-4 ${ }^{\circledR}$ (Truchet et al., 2015), where a new IFP cycle begins at each generation. This improves the statistics by a factor $\sqrt{M}$, although inter-cycle correlations may possibly occur (Truchet et al., 2015).

\subsection{Generalized IFP for $\alpha$-eigenvalue equations}

By resorting to the formal identification between the importance $I_{\alpha}$ of a neutron and the adjoint flux $\varphi_{\alpha}^{\dagger}\left(\mathbf{r}_{0}, \mathbf{v}_{0}\right)$, the extension of the IFP method to $\alpha$-eigenvalue equations is straightforward. The importance $I_{\alpha}$ can be again estimated by recording the descendants after $M$ latent generations for an ancestor injected into the system at $\mathbf{r}_{0}, \mathbf{v}_{0}$. In view of the considerations presented above, it is convenient to consider the cases $\alpha_{0}>0$ and $\alpha_{0}<0$ separately. When $\alpha_{0}>0$, the importance formally satisfies the modified transport equation (21): neutrons are promoted to the next generation only by fissions, but the average number of delayed neutrons for precursor family $j$ is lowered by a factor $\lambda_{j} /\left(\lambda_{j}+\alpha\right)<1$. Moreover, neutrons can be also captured by $\Sigma_{\alpha}$ during the latent generations, in which case they are removed and can not further contribute to the asymptotic importance.

When $\alpha_{0}<0$, the importance formally satisfies the modified transport equation (23): in this case, neutrons can be promoted to the next generation either by fissions (but the average number of delayed neutrons for precursor family $j$ is increased by a factor $\lambda_{j} /\left(\lambda_{j}+\alpha\right)>1$ ), or by the $\alpha$-production term in Eq. (14), associated to $v_{\eta}$ copy neutrons. Additionally, an absorption term $\Sigma_{\alpha, \eta}$ must be taken into account into the total cross section during transport in each generation.

The adjoint fundamental eigenmode $\varphi_{\alpha}^{\dagger}\left(\mathbf{r}_{0}, \mathbf{v}_{0}\right)$ is therefore estimated as above by recording the total number of descendants at generation $M+1$ for a single neutron starting at $\mathbf{r}_{0}, \mathbf{v}_{0}$. Contrary to the IFP algorithm for $k$-eigenvalue equation, knowledge of the fundamental $\alpha_{0}$ eigenvalue is required here, since the modified transport operators appearing in the $I_{\alpha}$ importance equation depend on $\alpha_{0}$. In practice, on can first apply the $\alpha-k$ power iteration to determine $\alpha_{0}$, ad then run fixed-source calculations based on the modified IFP algorithm (by keeping $\alpha_{0}$ fixed) to estimate $I_{\alpha}$ for a given initial neutron source. During the fixed-source calculations, the multiplication factor $k^{(g)}$ can be estimated at each generation, and eventually converges to $k^{(g)} \rightarrow 1$ for large $M$. The algorithm is schematically illustrated in Fig. 1 .

Bi-linear forms of the kind $\left\langle\varphi_{\alpha}^{\dagger}, A \varphi_{\alpha}\right\rangle$ can also be computed by the generalized IFP method. In particular, the adjoint-weighted kinetics parameters obtained by using the $\alpha$ eigenmode expansion emerging in reactor period calculations are defined as

$$
\Lambda_{\mathrm{eff}}^{\alpha}=\frac{\left\langle\varphi_{\alpha}^{\dagger}, \frac{1}{\mathrm{v}} \varphi_{\alpha}\right\rangle}{\left\langle\varphi_{\alpha}^{\dagger}, F \varphi_{\alpha}\right\rangle}, \quad \beta_{\mathrm{eff}, \mathrm{j}}^{\alpha}=\frac{\left\langle\varphi_{\alpha}^{\dagger}, F_{\mathrm{d}}^{j} \varphi_{\alpha}\right\rangle}{\left\langle\varphi_{\alpha}^{\dagger}, F \varphi_{\alpha}\right\rangle},
$$

respectively, with $\beta_{\mathrm{eff}}^{\alpha}=\sum_{j} \beta_{\mathrm{eff}, \mathrm{j}}^{\alpha}$. The associated in-hour equation reads

$$
\rho_{\alpha}=\alpha \Lambda_{\mathrm{eff}}^{\alpha}+\sum_{j} \beta_{\mathrm{eff}, \mathrm{j}}^{\alpha} \frac{\alpha}{\lambda_{j}+\alpha}
$$




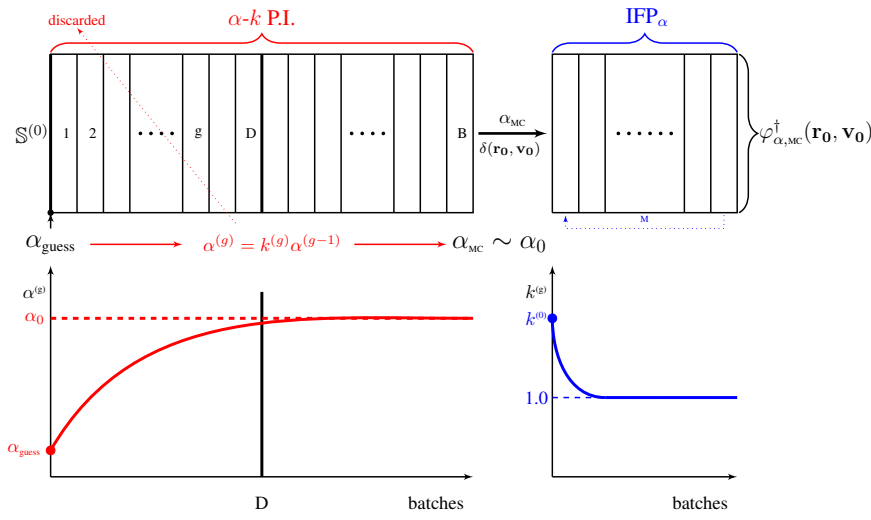

Figure 1: A schematic representation of the generalized IFP algorithm for the fundamental adjoint $\alpha$-eigenmode $\varphi_{\alpha}^{\dagger}\left(\mathbf{r}_{0}, \mathbf{v}_{0}\right)$. First, $\alpha$ is determined by running a $\alpha-k$ power iteration (P. I.), with arbitrary initial source $\mathcal{S}^{(0)}$, a large number $B$ of cycles, and $D$ discarded cycles. Then, fixed-source calculations based on the modified IFP algorithm (by keeping $\alpha$ fixed) are run to estimate $I_{\alpha} \propto \varphi_{\alpha}^{\dagger}\left(\mathbf{r}_{0}, \mathbf{v}_{0}\right)$ for a given initial source. The number of latent generations is $M$ : the multiplication factor $k^{(g)}$ can be estimated at each generation, and eventually converges to $k^{(g)} \rightarrow 1$ for large $M$.

$\rho_{\alpha}$ being the so-called dynamic reactivity of the system $\left({ }_{\text {Henry }}{ }^{250}\right.$ 1964). Such kinetics parameters can be computed by combining the $\alpha-k$ power iteration for $\varphi_{\alpha}$ with the generalized IFP al230 gorithm for $\varphi_{\alpha}^{\dagger}$. The $\alpha-k$ power iteration is first run until convergence is attained, and the neutron population is distributed on the fundamental eigenmode $\varphi_{\alpha}$. Then, at each successive generation $g$ a neutron is labelled as an ancestor. The corresponding importance $I_{\alpha}$ of this ancestor is estimated at a later generation $g+M+1$ via the generalized IFP method, depending on the sign of $\alpha_{0}$. Finally, the scalar products involved in Eq. 27) are computed by multiplying the score associated to the ancestor (pertaining to generation $g$ ) by its importance. The algorithm is ${ }^{260}$ schematically illustrated in Fig. 2 .

Total and delayed fission contributions can be computed from

$$
\begin{gathered}
\left\langle\varphi_{\alpha}^{\dagger}, F \varphi_{\alpha}\right\rangle \propto \sum_{i \neq i_{\alpha}}(\pi)_{i}, \\
\left\langle\varphi_{\alpha}^{\dagger}, F_{\mathrm{d}}^{j} \varphi_{\alpha}\right\rangle \propto \sum_{i \neq i_{\alpha}}\left(\frac{\lambda_{j}+\alpha}{\lambda_{j}}\right)_{i}\left(\pi_{\mathrm{d}, \mathrm{j}}\right)_{i},
\end{gathered}
$$

where we have excluded the contributions $i_{\alpha}$ to importance $(\pi)_{i}$ due to ancestors that have been promoted at a given generation $g$ by the $\alpha$-productions, and we have further rescaled each delayed fission term by a factor $\left(\lambda_{j}+\alpha\right) / \lambda_{j}$ in order to restore the correct weight to ancestors having being promoted to generation $g$ by a delayed fission. The role of the rescaling factor is analogous to that of the factor $1 / k$ for fission productions in the standard IFP method. In the $\alpha-k$ power iteration the multiplication factor is equal to $k=1$ once convergence is achieved, and thus does not explicitly appear in Eqs. (29).

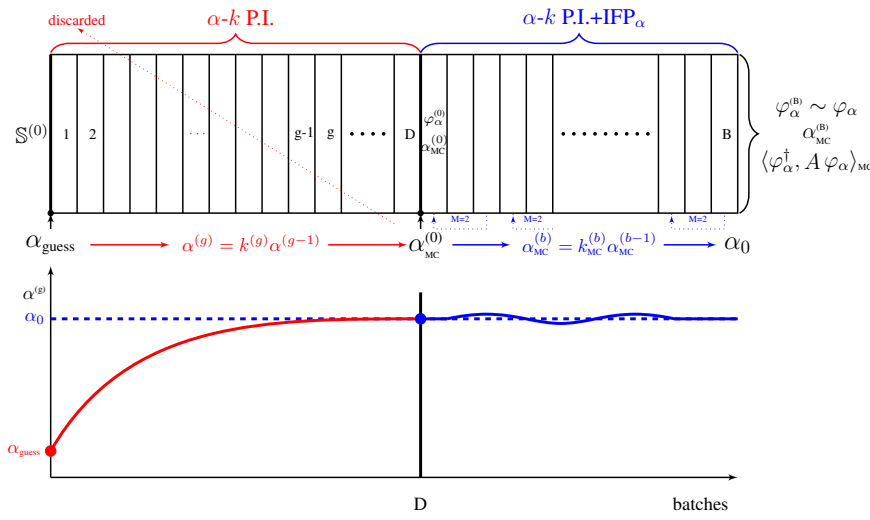

Figure 2: A schematic representation of the generalized IFP algorithm for bi-linear forms $\left\langle\varphi_{\alpha}^{\dagger}, A \varphi_{\alpha}\right\rangle$. The $\alpha$ - $k$ power iteration (P. I.) is first run until convergence is attained, starting from an arbitrary initial source $\mathcal{S}^{(0)}$ : after $D$ discarded cycles, neutrons will be distributed on the fundamental eigenmode $\varphi_{\alpha}$. Then, at each successive generation $g$ a neutron is labelled as an ancestor. The corresponding importance $I_{\alpha} \propto \varphi_{\alpha}^{\dagger}$ of this ancestor is estimated at a later generation $g+M+1$ via the generalized IFP method. Finally, the scalar product is computed by multiplying the score associated to the ancestor by its importance.

\section{Verification on a simple multiplying system}

The generalized IFP algorithm has been integrated in a development version of the TRIPOLI-4 ${ }^{\circledR}$ Monte Carlo code (Brun et al. 2015) developed at CEA, by suitably modifying the existing routines previously conceived for the standard IFP methods. The proposed methods need a thorough verification: to this aim, we analyze here a simple, yet non trivial, benchmark reactor configuration consisting of an infinite medium with two energy groups. For this model, exact reference solutions can be easily derived for the (direct and adjoint) flux, the kinetics parameters, and the reactivity (Kiedrowski, 2010).

\subsection{Analytical solutions}

Let us consider a homogeneous system of infinite size, with two energy groups $\mathrm{v}_{1}$ (fast) and $\mathrm{v}_{2}$ (thermal) and two delayed families $a$ and $b$. Specifications are basically taken from the model proposed in (Kiedrowski, 2010). We assume that no upscattering is possible, fissions can be induced only from neutrons colliding in the thermal group $g=2$ and finally that all fission neutrons are emitted exclusively in $g=1$. Under such conditions the $k$-eigenvalue transport problem can be reduced to a system of two equations for the scalar flux $\varphi_{k}$, namely,

$$
\begin{aligned}
& \Sigma_{r, 1} \varphi_{k, 1}=\frac{1}{k_{\mathrm{eff}}}\left(1-\beta_{\mathrm{tot}}+\xi_{1}\right) \nu_{\mathrm{f}, 2} \Sigma_{\mathrm{f}, 2} \varphi_{k, 2} \\
& \Sigma_{r, 2} \varphi_{k, 2}=\Sigma_{s, 12} \varphi_{k, 1}+\frac{1}{k} \xi_{2} v_{\mathrm{f}, 2} \Sigma_{\mathrm{f}, 2} \varphi_{k, 2},
\end{aligned}
$$

where $\varphi_{k, g}=\varphi_{k}\left(\mathrm{v}_{g}\right)$ and $\Sigma_{x, g}=\Sigma_{x}\left(\mathrm{v}_{g}\right) . \Sigma_{s, g i}=\Sigma_{s}\left(\mathrm{v}_{g} \rightarrow \mathrm{v}_{i}\right)$ is the differential scattering kernel, $\Sigma_{r, g}=\Sigma_{t, g}-\Sigma_{s, g g}$ the removal cross-section of group $g, \Sigma_{\mathrm{f}, g}$ the fission cross-section of group 
$g, v_{\mathrm{f}, g}$ the number of neutrons produced by a fission in group $g, \chi_{j, g}$ is the delayed neutron spectrum from precursor family $j$ to energy group $g, \beta_{j}$ the delayed neutron fraction of family $j$, $\beta_{\text {tot }}=\beta_{a}+\beta_{b}$, and $\xi_{g}=\chi_{a, g} \beta_{a}+\chi_{b, g} \beta_{b}$. The associated adjoint equations can be obtained from Eqs. 30 from transposition, i.e.,

$$
\begin{aligned}
& \Sigma_{r, 1} \varphi_{k, 1}^{\dagger}=\Sigma_{s, 12} \varphi_{k, 2}^{\dagger} \\
& \Sigma_{r, 2} \varphi_{k, 2}^{\dagger}=\frac{1}{k}\left[\left(1-\beta+\xi_{1}\right) v_{\mathrm{f}, 2} \Sigma_{\mathrm{f}, 2} \varphi_{k, 1}^{\dagger}+\xi_{2} v_{\mathrm{f}, 2} \Sigma_{\mathrm{f}, 2} \varphi_{k, 2}^{\dagger}\right] .
\end{aligned}
$$

After some algebra, the following analytical solutions can be obtained. From Eqs. 30, we have the fundamental eigenvalue

$$
k_{0}=\frac{v_{\mathrm{f}, 2} \Sigma_{\mathrm{f}, 2}}{\Sigma_{r, 2}}\left[z^{\dagger}\left(1-\beta+\xi_{1}\right)+\xi_{2}\right],
$$

with the associated reactivity $\rho_{k}=\left(k_{0}-1\right) / k_{0}$, and the critical flux ratio for $\varphi_{k, g}$

$$
z=\frac{\varphi_{k, 2}}{\varphi_{k, 1}}=\frac{\Sigma_{s, 12}}{\Sigma_{r, 2}-\frac{1}{k_{0}} \xi_{2} v_{\mathrm{f}, 2} \Sigma_{\mathrm{f}, 2}} .
$$

From Eqs. 30 we have the adjoint critical flux ratio for $\varphi_{k, g}^{\dagger}$

$$
z^{\dagger}=\frac{\varphi_{k, 1}^{\dagger}}{\varphi_{k, 2}^{\dagger}}=\frac{\Sigma_{s, 12}}{\Sigma_{r, 1}}
$$

Based on these results, the adjoint-weighted parameters yield (Kiedrowski 2010)

$$
\beta_{\mathrm{eff}, \mathrm{j}}=\frac{\left\langle\varphi_{k}^{\dagger}, F_{d}^{j} \varphi_{k}\right\rangle}{\left\langle\varphi_{k}^{\dagger}, F \varphi_{k}\right\rangle}=\frac{z^{\dagger} \chi_{1}^{j} \beta_{j}+\chi_{2}^{j} \beta_{j}}{z^{\dagger}\left(1-\beta+\xi_{1}\right)+\xi_{2}},
$$

with

$$
\beta_{\mathrm{eff}}=\sum_{j} \beta_{\mathrm{eff}, \mathrm{j}}=\frac{z^{\dagger} \xi_{1}+\xi_{2}}{z^{\dagger}\left(1-\beta+\xi_{1}\right)+\xi_{2}},
$$

and

$$
\Lambda_{\mathrm{eff}}=\frac{\left\langle\varphi_{k}^{\dagger}, \frac{1}{\mathrm{v}} \varphi_{k}\right\rangle}{\left\langle\varphi_{k}^{\dagger}, F \varphi_{k}\right\rangle}=\frac{\frac{1}{\mathrm{v}_{1}} \frac{z^{\dagger}}{z}+\frac{1}{\mathrm{v}_{2}}}{v_{\mathrm{f}, 2} \Sigma_{\mathrm{f}, 2}\left[z^{\dagger}\left(1-\beta+\xi_{1}\right)+\xi_{2}\right]} .
$$

As for the $\alpha$-eigenmode equations, we obtain

$$
\begin{aligned}
\frac{\alpha}{\mathrm{v}_{1}} \varphi_{\alpha, 1}+\varphi_{\alpha, 1} & =\left(1-\beta+\zeta_{1}\right) \nu_{\mathrm{f}, 2} \Sigma_{\mathrm{f}, 2} \varphi_{\alpha, 2} \\
\frac{\alpha}{\mathrm{v}_{2}} \varphi_{\alpha, 2}+\Sigma_{r, 2} \varphi_{\alpha, 2} & =\Sigma_{s, 12} \varphi_{\alpha, 1}+\zeta_{2} v_{\mathrm{f}, 2} \Sigma_{\mathrm{f}, 2} \varphi_{\alpha, 2},
\end{aligned}
$$

where we have set

$$
\zeta_{i}=\sum_{j} \frac{\lambda_{j}}{\lambda_{j}+\alpha} \chi_{d}^{j}\left(\mathrm{v}_{i}\right) \beta_{j}=\xi_{i}-\sum_{j} \frac{\alpha}{\lambda_{j}+\alpha} \chi_{d}^{j}\left(\mathrm{v}_{i}\right) \beta_{j},
$$

and $\lambda_{j}$ is the precursor decay constant for family $j$. By transposition of Eqs. (38), the adjoint equations read

$$
\begin{aligned}
& \frac{\alpha}{\mathrm{v}_{1}} \varphi_{\alpha, 1}^{\dagger}+\Sigma_{r, 1} \varphi_{\alpha, 1}^{\dagger}=\Sigma_{s, 12} \varphi_{\alpha, 2}^{\dagger} \\
& \left(1-\beta+\zeta_{1}\right) v_{\mathrm{f}, 2} \Sigma_{\mathrm{f}, 2} \varphi_{\alpha, 1}^{\dagger}+\frac{\alpha}{\mathrm{v}_{2}} \varphi_{\alpha, 2}^{\dagger}+\Sigma_{r, 2} \varphi_{\alpha, 2}^{\dagger}=\zeta_{2} v_{\mathrm{f}, 2} \Sigma_{\mathrm{f}, 2} \varphi_{\alpha, 2}^{\dagger} .
\end{aligned}
$$

\begin{tabular}{cccccccc}
\hline$g$ & $\mathrm{v}$ & $\Sigma_{a, g}$ & $\Sigma_{\mathrm{f}, g}$ & $\chi_{a \rightarrow g}$ & $\chi_{b \rightarrow g}$ & $\Sigma_{\mathrm{s}, g \rightarrow 1}$ & $\Sigma_{\mathrm{s}, g \rightarrow 2}$ \\
\hline 1 & 10 & 1 & 0 & $3 / 4$ & $1 / 2$ & $1 / 2$ & $1 / 2$ \\
2 & 5 & 1 & 1 & $1 / 4$ & $1 / 2$ & 0 & 1 \\
\hline
\end{tabular}

Table 1: Reference values for the physical parameters of the two-group infinite medium system.

The fundamental $\alpha_{0}$ eigenvalue can be obtained from (analytically or numerically) solving the fourth-order polynomial equation stemming from Eqs. (38), namely,

$$
c_{4} \alpha^{4}+c_{3} \alpha^{3}+c_{2} \alpha^{2}+c_{1} \alpha+c_{0}=0
$$

whose coefficients are explicitly provided in Appendix A and singling out the root with the largest real part. The direct flux ratio $z_{\alpha}$ can then be computed from Eqs. (38), i.e.,

$$
z_{\alpha}=\frac{\varphi_{\alpha, 2}}{\varphi_{\alpha, 1}}=\frac{\Sigma_{s, 12}}{\frac{\alpha_{0}}{\mathrm{v}_{2}}+\Sigma_{r, 2}-\zeta_{2} v_{\mathrm{f}, 2} \Sigma_{\mathrm{f}, 2}},
$$

and the adjoint flux ratio $z_{\alpha}^{\dagger}$ can be similarly computed from Eqs. 40, and yields

$$
z_{\alpha}^{\dagger}=\frac{\varphi_{\alpha, 1}^{\dagger}}{\varphi_{\alpha, 2}^{\dagger}}=\frac{\Sigma_{s, 12}}{\frac{\alpha_{0}}{v_{1}}+\Sigma_{r, 1}} .
$$

Based on these expressions, the adjoint-weighted kinetics parameters $\beta_{\alpha}$ and $\Lambda_{\alpha}$ can be computed explicitly, and read

$$
\beta_{\mathrm{eff}, \mathrm{j}}^{\alpha}=\frac{\left\langle\varphi_{\alpha}^{\dagger}, F_{d}^{j} \varphi_{\alpha}\right\rangle}{\left\langle\varphi_{\alpha}^{\dagger}, F \varphi_{\alpha}\right\rangle}=\frac{z_{\alpha}^{\dagger} \chi_{1}^{j} \beta_{j}+\chi_{2}^{j} \beta_{j}}{z_{\alpha}^{\dagger}\left(1-\beta+\xi_{1}\right)+\xi_{2}},
$$

$$
\beta_{\mathrm{eff}}^{\alpha}=\sum_{j} \beta_{\alpha, j}=\frac{z_{\alpha}^{\dagger} \xi_{1}+\xi_{2}}{z_{\alpha}^{\dagger}\left(1-\beta+\xi_{1}\right)+\xi_{2}},
$$

$$
\Lambda_{\mathrm{eff}}^{\alpha}=\frac{\left\langle\varphi_{\alpha}^{\dagger}, \frac{1}{\mathrm{v}} \varphi_{\alpha}\right\rangle}{\left\langle\varphi_{\alpha}^{\dagger}, F \varphi_{\alpha}\right\rangle}=\frac{\frac{1}{\mathrm{v}_{1}} \frac{z_{\alpha}^{\dagger}}{z_{\alpha}}+\frac{1}{\mathrm{v}_{2}}}{v_{\mathrm{f}, 2} \Sigma_{\mathrm{f}, 2}\left[z_{\alpha}^{\dagger}\left(1-\beta+\xi_{1}\right)+\xi_{2}\right]},
$$

Observe that the direct and adjoint flux ratios, and hence also the kinetics parameters, depend on the fundamental eigenvalue $\alpha_{0}$. Finally, the dynamic reactivity $\rho_{\alpha}$ can be obtained from the in-hour formula in Eq. 28.

\subsection{Setting the model parameters}

The reference physical constants of the problem (in arbitrary units) have been set as reported in Tab. 1 Numerical values are basically taken from the specifications suggested in (Kiedrowski, 2010).

The delayed neutron fractions are $\beta_{a}=1 / 4, \beta_{b}=1 / 8$. With these parameters, the reactor is critical when $v_{\mathrm{f}, 2}=24 / 5$. The precursor decay constants do not affect the criticality condition: for our calculations, we have set $\lambda_{a}=5$ and $\lambda_{b}=7$. In the Monte Carlo simulations illustrated in the following, we have perturbed the reference critical configuration by making the system sub- or super-critical. 


\subsection{Computing the adjoint flux}

As a first verification test, we have computed the adjoint fundamental mode for the $\alpha$-eigenvalue equations. Since such equations are homogeneous, the normalization is arbitrary, and it is convenient to compute the ratio $z_{\alpha}^{\dagger}=\left(\varphi_{\alpha, 1}^{\dagger} / \varphi_{\alpha, 2}^{\dagger}\right)$.

As mentioned in the previous section, the $\alpha_{0}$ eigenvalue is needed in order to determine the adjoint fundamental mode with the generalized IFP method. In practice, one can first run a $\alpha-k$ power iteration, and estimate $\alpha_{0}$; then, run the generalized IFP algorithm with fixed $\alpha_{0}$, and determine the adjoint flux as detailed above. The convergence of the $\alpha$ - $k$ power iteration is slow, and requires about $10^{2}$ cycles to convergence even for this simple two-group system. The convergence of the generalized IFP method is much faster, and takes about $M=10$ latent generations, which is comparable to the number of latent generations required by the standard IFP method for $k$-eigenvalue equations. For the generalized IFP method we have run $2 \times 10^{3}$ batches with $5 \cdot 10^{5}$ neutrons per batch.

The system has been made sub-critical by taking $v_{\mathrm{f}, 2}=20 / 5$ (which would correspond to $k_{\mathrm{eff}}=0.83333$ ) and super-critical by taking $v_{\mathrm{f}, 2}=28 / 5$ (which would correspond to $k_{\mathrm{eff}}=$ 1.16707). The corresponding simulation results are shown in Tab. 2. an excellent agreement is found with respect to the exact values given in Eq. (43).

\subsection{Computing the kinetics parameters}

Adjoint-weighted kinetics parameters have also been estimated by using the generalized IFP algorithm that has been plugged into an existing $\alpha-k$ power iteration scheme. At first, convergence to the fundamental direct $\alpha$-eigenmode is attained by running a sufficiently large number of cycles. Then, the generalized IFP method is used at each cycle so as to compute the adjoint-weighted scores (the value of $\alpha_{0}$ is known after reaching equilibrium). For our calculations, 300 inactive cycles before convergence were discarded, and we ran $3 \times 10^{3}$ active cycles with $5 \times 10^{4}$ neutrons per cycle. We set $M=15$ latent generations. The comparison between Monte Carlo simulation results and the exact results derived in the previous sections is illustrated in Tab. 3 for a sub- and super-critical configuration obtained by setting $v_{\mathrm{f}, 2}=20 / 5$ and $v_{\mathrm{f}, 2}=28 / 5$, respectively. The simulation findings are in excellent agreement with the exact results derived above.

\subsection{Comparison to standard IFP}

As a final verification, we have computed the kinetics parameters and the associated dynamic reactivity $\rho_{\alpha}$ by varying the absorption cross section $\Sigma_{a, 1}$ close to the reference value $\Sigma_{a, 1}=$ 1 that makes the system exactly critical for $v_{\mathrm{f}, 2}=24 / 5$. Monte Carlo simulation results using the generalized IFP method are ${ }^{340}$ 政 respect to the exact formulas. In order to illustrate the difference that may arise from the choice of the eigenmode expansion, in the same figure we also display the kinetics parameters obtained by using the regular IFP method in a standard power iteration, i.e., by weighting with the fundamental $k$-eigenmode.

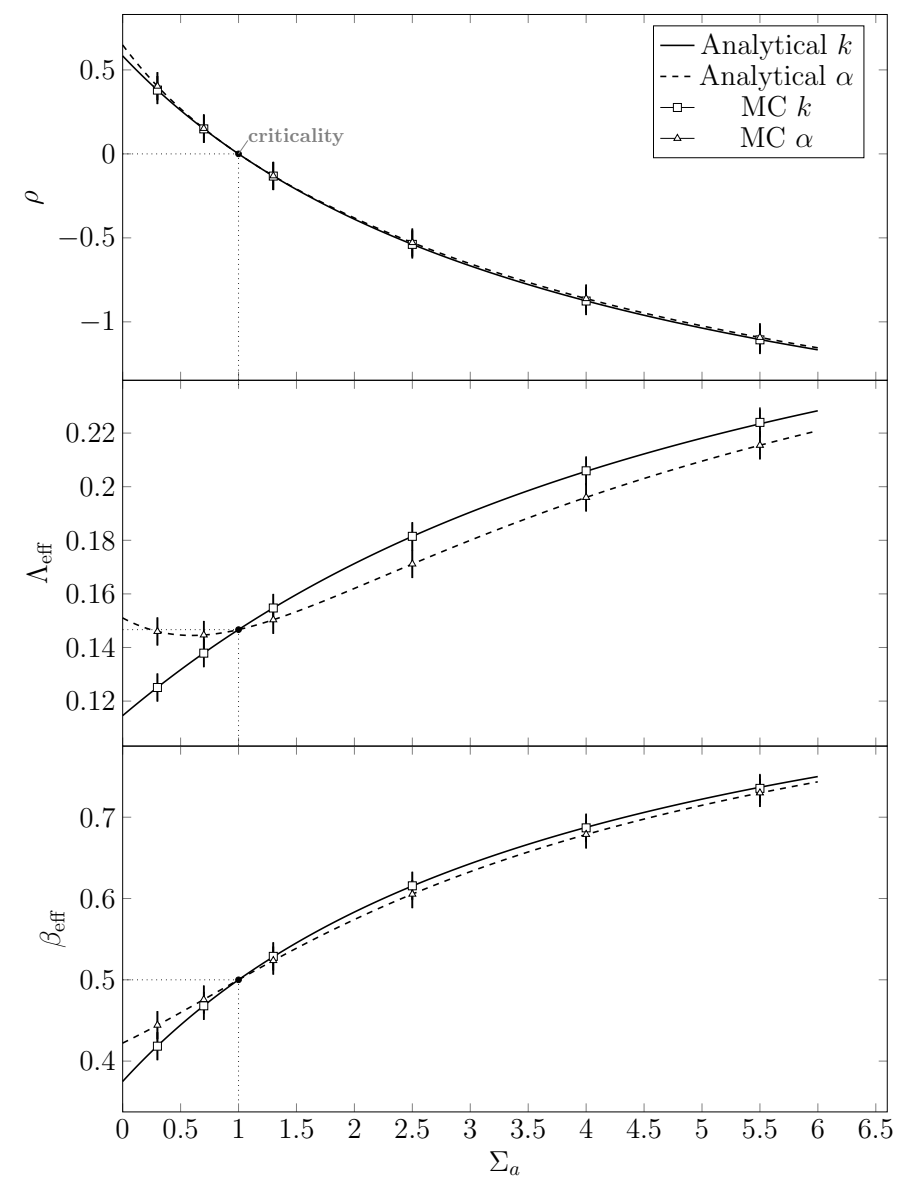

Figure 3: Adjoint-weighted kinetics parameters and reactivity for the two-group infinite medium reactor. Exact solutions are displayed as solid lines (corresponding to $k$ eigenvalue expansion) and dashed lines (corresponding to $\alpha$ eigenvalue expansion). Monte Carlo simulation results are displayed as symbols: squares for regular IFP method (corresponding to weighting by $I_{k}$ ), and triangles for the generalized IFP method (corresponding to weighting by $I_{\alpha}$ ). The cross section $\Sigma_{a, 1}$ is varied around the value $\Sigma_{a, 1}=1$ that makes the system exactly critical (see Tab. 1).

Monte Carlo simulation results are compared to the exact formulas. As expected, the two eigenmode decompositions coincide for $k_{0}=1$ and $\alpha_{0}=0$, and yield different results for the adjoint-weighted kinetics parameters otherwise. For the sake of completeness, in Fig. 3 we also contrast the dynamic reactivity $\rho_{\alpha}$, which is derived from the in-hour equation by injecting the kinetics parameters and the fundamental $\alpha_{0}$ eigenvalue, and the so-called static reactivity $\rho_{k}=\left(k_{0}-1\right) / k_{0}$, which depends on the fundamental $k$ eigenvalue. The values for $\rho_{\alpha}$ and $\rho_{k}$ differ away from the critical point, and the discrepancy is stronger in the super-critical than in the sub-critical regime.

\section{Application to reactor configurations}

In order to better apprehend the impact of choosing the $\alpha$ eigenmode expansion instead of the $k$-eigenmode expansion to 


\begin{tabular}{ccccc}
\hline$v_{\mathrm{f}, 2}$ & $\alpha_{0}$ & $\alpha_{M C}$ & $z_{\alpha}^{\dagger}$ & $\left(\varphi_{\alpha, 1}^{\dagger} / \varphi_{\alpha, 2}^{\dagger}\right)_{M C}$ \\
\hline $28 / 5$ & 0.68797 & $0.68789 \pm 6.6 \times 10^{-5}$ & 0.31872 & $0.31892 \pm 1.4 \times 10^{-4}$ \\
\hline $20 / 5$ & -0.73138 & $-0.73128 \pm 6.6 \times 10^{-5}$ & 0.35042 & $0.35025 \pm 1.4 \times 10^{-4}$ \\
\hline
\end{tabular}

Table 2: Adjoint flux ratios for sub -and super-critical configurations of the two-group system.

For all the multiplying systems considered here, the kinetics parameters agree with each other within one standard deviation, and no systematic discrepancy can be detected between the results coming from the generalized IFP method and those coming from the standard IFP method. This is expected, since these configurations are almost exactly critical and the $\alpha$ or $k$ -

compute the kinetics parameters in more realistic systems, we have selected some reactor configurations with simple geometry and material compositions, and yet non trivial flux shape. We will adopt the JEFF-3.1.1 nuclear data library, where precursors have been regrouped into 8 families with identical decay constants for all nuclides (Santamarina et al. 2009).

As a first case we have examined Godiva, a bare sphere of highly enriched metallic Uranium (OECD NEA, 2010). Specifications for the Monte Carlo model were taken from those of HEU-MET-FAST-001 in NEA/NSC/DOC(95)03/II (Mosteller and Kiedrowski, 2011; OECD NEA, 2010). Based on the algorithms detailed above, we have computed both $k$ and $\alpha$-adjointweighted kinetics parameters with TRIPOLI- $4{ }^{\circledR}$ : the simulation results are reported in Tab. 4 for $10^{3}$ inactive cycles, $5 \times 10^{3}$ active cycles and $5 \times 10^{4}$ neutrons per cycle. The number of latent generations was set to $M=20$.

The second case is Thor, a Thorium-reflected Plutonium sphere (OECD NEA, 2010). Specifications for the Monte Carlo model were taken from those of PU-MET-FAST-008 in NEA/NSC/DOC(95)03/I (Mosteller and Kiedrowski, 2011, OECD NEA, 2010). The simulation results obtained with TRIPOLI- $4{ }^{\circledR}$ are reported in Tab. 5 for $10^{3}$ inactive cycles, $5 \times 10^{3^{395}}$ active cycles and $5 \times 10^{4}$ neutrons per cycle. The number of latent generations was set to $M=20$.

We conclude by considering the case of IPEN/MB-01, a zero power critical facility designed for the measurement of reactor ${ }^{400}$ physics parameters. The facility consists of a $28 \times 26$ rectangular array of $\mathrm{UO}_{2}$ fuel rods $4.3 \%$ enriched and clad by stainless steel (SS-304) inside a light water tank (see Fig. 4). The control banks are composed by $12 \mathrm{Ag}-\mathrm{In}$-Cd rods and the safety banks by $12 \mathrm{~B}_{4} \mathrm{C}$ rods. The pitch of the IPEN/MB- $01^{405}$ reactor was chosen to be close to the optimum moderator ratio. The specifications for the IPEN/MB-01 configuration are taken from case LEU-COMP-THERM-077 of NEA/NSC/DOC (95)03/I, as provided in (dos Santos and Diniz, 2014, dos Santos et al. 2013) and references therein. The simulation results ${ }^{410}$ obtained with TRIPOLI- $4{ }^{\circledR}$ are reported in Tab. 6 for $10^{2}$ inactive cycles, $4 \times 10^{3}$ active cycles and $10^{5}$ neutrons per cycle. The number of latent generations was set to $M=20$. eigenvalue expansions must yield similar results.

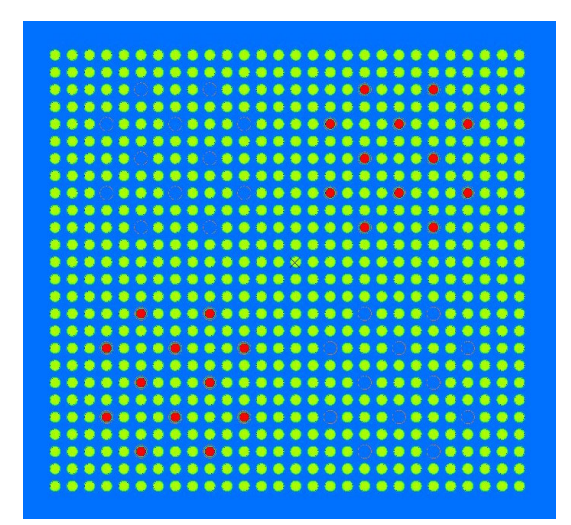

Figure 4: (Color online) Radial view of the TRIPOLI- $4{ }^{\circledR}$ model of the IPEN/MB-01 core.

\section{Conclusions}

In this paper we have proposed a generalized IFP method that enables the computation of the adjoint fundamental $\alpha$-eigenmode and of arbitrary scores weighted by the $\alpha$ importance. We have shown that this algorithm can be easily implemented in production Monte Carlo codes by suitably modifying existing standard IFP routines. The generalized IFP method has been used to compute the $\alpha$-adjoint-weighted kinetics parameters, which might be of interest in reactor period calculations.

Future research will be aimed at investigating the effects of nuclear data and reactor geometry on the reactivity and on the kinetics parameters (as obtained by either the $k$ or $\alpha$ eigenvalue expansion) close to criticality.

Although in this work we have focused exclusively on the application to bi-linear forms of the kind $\left\langle\varphi_{\alpha}^{\dagger}, A \varphi_{\alpha}\right\rangle$, it is worth mentioning that the proposed strategy could be more broadly extended to the calculation of $\left\langle\varphi_{k}^{\dagger}, A \varphi_{\alpha}\right\rangle$ or $\left\langle\varphi_{\alpha}^{\dagger}, A \varphi_{k}\right\rangle$ by plugging the standard IFP method into the $\alpha-k$ power iteration or by plugging the generalized IFP method into the regular power iteration, respectively. These expressions appear for instance in perturbative approaches for reactivity calculations (Nauchi, 2014).

\section{Appendix A. The coefficients of Eq. (41)}

The coefficients of Eq. (41) can be explicitly derived by inspection of the system (38). The expressions are reported here for the sake of completeness. This yields $c_{4}=1$ from normalization,

$$
c_{3}=\lambda_{a}+\lambda_{b}+\Sigma_{r, 2} \mathrm{v}_{2}+\Sigma_{r, 1} \mathrm{v}_{1}
$$




\begin{tabular}{lccccc}
\hline & \multicolumn{2}{c}{$v_{\mathrm{f}, 2}=28 / 5$} & & \multicolumn{2}{c}{$v_{\mathrm{f}, 2}=20 / 5$} \\
\cline { 2 - 3 } \cline { 5 - 6 } & & & & \\
& Analytical & Monte Carlo & & Analytical & Monte Carlo \\
\hline$\alpha_{0}$ & 0.68797 & $0.68744 \pm 8.6 \times 10^{-5}$ & & -0.73138 & $-0.73095 \pm 8.6 \times 10^{-5}$ \\
$\Lambda_{\mathrm{eff}}^{\alpha}$ & 0.13102 & $0.13101 \pm 1.6 \times 10^{-5}$ & & 0.16787 & $0.16780 \pm 2.7 \times 10^{-5}$ \\
$\beta_{\mathrm{eff}}^{\alpha}$ & 0.50679 & $0.50680 \pm 8.2 \times 10^{-5}$ & & 0.49258 & $0.49269 \pm 8.6 \times 10^{-5}$ \\
$\beta_{\mathrm{eff}, a}^{\alpha}$ & 0.30272 & $0.30269 \pm 7.8 \times 10^{-5}$ & & 0.29703 & $0.29717 \pm 7.9 \times 10^{-5}$ \\
$\beta_{\mathrm{eff}, b}^{\alpha}$ & 0.20407 & $0.20411 \pm 6.7 \times 10^{-5}$ & & 0.19555 & $0.19552 \pm 6.9 \times 10^{-5}$ \\
$\rho_{\alpha}$ & 0.14501 & $0.14490 \pm 2.2 \times 10^{-5}$ & & -0.19649 & $-0.19634 \pm 3.5 \times 10^{-5}$ \\
\hline
\end{tabular}

Table 3: Adjoint-weighted kinetics parameters for a two-group system in super- and sub-critical configurations.

\begin{tabular}{lcc}
\hline & $\left\langle\varphi_{k}^{\dagger}, \bullet\right\rangle$ & $\left\langle\varphi_{\alpha}^{\dagger}, \bullet\right\rangle$ \\
\hline$\Lambda_{\text {eff }}$ & $5.702 \pm 0.003$ & $5.733 \pm 0.003$ \\
$\beta_{\text {eff }}$ & $645.01 \pm 2.98$ & $645.78 \pm 2.81$ \\
$\beta_{1, \text { eff }}$ & $24.19 \pm 0.58$ & $23.46 \pm 0.20$ \\
$\beta_{2, \text { eff }}$ & $89.16 \pm 1.10$ & $91.79 \pm 0.91$ \\
$\beta_{3, \text { eff }}$ & $66.53 \pm 0.95$ & $66.03 \pm 0.82$ \\
$\beta_{4, \text { eff }}$ & $128.15 \pm 1.34$ & $128.22 \pm 1.31$ \\
$\beta_{5, \text { eff }}$ & $200.40 \pm 1.67$ & $199.79 \pm 1.67$ \\
$\beta_{6, \text { eff }}$ & $61.52 \pm 0.94$ & $63.28 \pm 0.95$ \\
$\beta_{7, \text { eff }}$ & $58.47 \pm 0.91$ & $56.94 \pm 0.89$ \\
$\beta_{8, \text { eff }}$ & $16.59 \pm 0.48$ & $16.27 \pm 0.49$ \\
\hline
\end{tabular}

Table 4: Comparison of kinetics parameters as computed by using the standard IFP method (left) and the generalized IFP method (right). The case of Godiva. The generation time is expressed in nanoseconds and the delayed neutron fractions are expressed in pcm.

\begin{tabular}{lcc}
\hline Parameter & $\left\langle\varphi_{k}^{\dagger}, \bullet\right\rangle$ & $\left\langle\varphi_{\alpha}^{\dagger}, \bullet\right\rangle$ \\
\hline$\Lambda_{\text {eff }}$ & $10.34 \pm 0.01$ & $10.31 \pm 0.01$ \\
$\beta_{\text {eff }}$ & $214.50 \pm 1.62$ & $210.53 \pm 2.15$ \\
$\beta_{1, \mathrm{eff}}$ & $6.38 \pm 0.28$ & $5.90 \pm 0.61$ \\
$\beta_{2, \mathrm{eff}}$ & $44.98 \pm 0.76$ & $44.65 \pm 1.27$ \\
$\beta_{3, \mathrm{eff}}$ & $21.04 \pm 0.51$ & $21.08 \pm 0.78$ \\
$\beta_{4, \mathrm{eff}}$ & $31.63 \pm 0.63$ & $30.19 \pm 0.71$ \\
$\beta_{5, \mathrm{eff}}$ & $74.03 \pm 0.94$ & $73.14 \pm 1.02$ \\
$\beta_{6, \mathrm{eff}}$ & $11.35 \pm 0.37$ & $10.70 \pm 0.38$ \\
$\beta_{7, \mathrm{eff}}$ & $21.03 \pm 0.52$ & $20.63 \pm 0.51$ \\
$\beta_{8, \mathrm{eff}}$ & $4.06 \pm 0.23$ & $4.25 \pm 0.22$ \\
\hline
\end{tabular}

Table 5: Comparison of kinetics parameters as computed by using the standard IFP method (left) and the generalized IFP method (right). The case of Thor. The generation time is expressed in nanoseconds and the delayed neutron fractions are expressed in pcm. 


\begin{tabular}{lcc}
\hline Parameter & $\left\langle\varphi_{k}^{\dagger}, \bullet\right\rangle$ & $\left\langle\varphi_{\alpha}^{\dagger}, \bullet\right\rangle$ \\
\hline$\Lambda_{\text {eff }}$ & $30.50 \pm 0.01$ & $30.49 \pm 0.01$ \\
$\beta_{\text {eff }}$ & $777.35 \pm 2.22$ & $776.44 \pm 2.43$ \\
$\beta_{1, \text { eff }}$ & $24.58 \pm 0.40$ & $24.91 \pm 0.61$ \\
$\beta_{2, \text { eff }}$ & $115.43 \pm 0.87$ & $114.04 \pm 1.06$ \\
$\beta_{3, \text { eff }}$ & $69.55 \pm 0.67$ & $68.53 \pm 0.77$ \\
$\beta_{4, \text { eff }}$ & $149.30 \pm 0.97$ & $147.44 \pm 1.05$ \\
$\beta_{5, \text { eff }}$ & $251.54 \pm 1.26$ & $252.03 \pm 1.31$ \\
$\beta_{6, \text { eff }}$ & $78.14 \pm 0.71$ & $79.15 \pm 0.71$ \\
$\beta_{7, \text { eff }}$ & $65.34 \pm 0.66$ & $67.51 \pm 0.66$ \\
$\beta_{8, \text { eff }}$ & $23.46 \pm 0.39$ & $22.82 \pm 0.38$ \\
\hline
\end{tabular}

Table 6: Comparison of kinetics parameters as computed by using the standard IFP method (left) and the generalized IFP method (right). The case of IPEN/MB-01. The generation time is expressed in microseconds and the delayed neutron fractions are expressed in $\mathrm{pcm}$.

$$
\begin{gathered}
c_{2}=\lambda_{a} \lambda_{b}+\left(\Sigma_{r, 2} \mathrm{v}_{2}+\Sigma_{r, 1} \mathrm{v}_{1}\right)\left(\lambda_{a}+\lambda_{b}\right) \\
-(1-\beta) v_{\mathrm{f}, 2} \Sigma_{\mathrm{f}, 2} \Sigma_{s, 12} \mathrm{v}_{2} \mathrm{v}_{1}+\Sigma_{r, 2} \Sigma_{r, 1} \mathrm{v}_{2} \mathrm{v}_{1} \\
-v_{\mathrm{f}, 2} \Sigma_{\mathrm{f}, 2} \mathrm{v}_{1}\left(\chi_{1}^{a} \beta_{a} \lambda_{a}+\chi_{1}^{b} \beta_{b} \lambda_{b}\right),
\end{gathered}
$$

$$
\begin{gathered}
c_{1}=\lambda_{a} \lambda_{b}\left(\Sigma_{r, 2} \mathrm{v}_{2}+\Sigma_{r, 1} \mathrm{v}_{1}\right)-(1-\beta) v_{\mathrm{f}, 2} \Sigma_{\mathrm{f}, 2} \Sigma_{s, 12} \mathrm{v}_{2} \mathrm{v}_{1}\left(\lambda_{a}+\lambda_{b}\right) \\
+\Sigma_{r, 2} \Sigma_{r, 1} \mathrm{v}_{2} \mathrm{v}_{1}\left(\lambda_{a}+\lambda_{b}\right) \\
-v_{\mathrm{f}, 2} \Sigma_{\mathrm{f}, 2} \Sigma_{s, 12} \mathrm{v}_{2} \mathrm{v}_{1}\left(\chi_{2}^{a} \beta_{a} \lambda_{a}+\chi_{2}^{b} \beta_{b} \lambda_{b}\right) \\
-v_{\mathrm{f}, 2} \Sigma_{\mathrm{f}, 2} \Sigma_{r, 2} \mathrm{v}_{2} \mathrm{v}_{1}\left(\chi_{1}^{a} \beta_{a} \lambda_{a}+\chi_{1}^{b} \beta_{b} \lambda_{b}\right) \\
-\lambda_{a} \lambda_{b} v_{\mathrm{f}, 2} \Sigma_{\mathrm{f}, 2} \mathrm{v}_{1}\left(\chi_{1}^{a} \beta_{a}+\chi_{1}^{b} \beta_{b}\right)
\end{gathered}
$$

and

$$
\begin{gathered}
c_{0}=\lambda_{a} \lambda_{b} \Sigma_{r, 2} \Sigma_{r, 1} \mathrm{v}_{2} \mathrm{v}_{1}-(1-\beta) \lambda_{a} \lambda_{b} \nu_{\mathrm{f}, 2} \Sigma_{\mathrm{f}, 2} \Sigma_{s, 12} \mathrm{v}_{2} \mathrm{v}_{1} \\
-\lambda_{a} \lambda_{b} v_{\mathrm{f}, 2} \Sigma_{\mathrm{f}, 2} \Sigma_{s, 12} \mathrm{v}_{2} \mathrm{v}_{1}\left(\chi_{2}^{a} \beta_{a}+\chi_{2}^{b} \beta_{b}\right) \\
-\lambda_{a} \lambda_{b} v_{\mathrm{f}, 2} \Sigma_{\mathrm{f}, 2} \Sigma_{r, 2} \mathrm{v}_{2} \mathrm{v}_{1}\left(\chi_{1}^{a} \beta_{a}+\chi_{1}^{b} \beta_{b}\right) .
\end{gathered}
$$

\section{Acknowledgements}

TRIPOLI ${ }^{\circledR}$ and TRIPOLI- $4{ }^{\circledR}$ are registered trademarks of CEA. The authors wish to thank Électricite de France (EDF) for partial financial support. A. Zoia and N. Terranova express 460 their gratitude to Dr. F. Malvagi of CEA/Saclay for fruitful discussions.

\section{References}

Bell, G. I., Glasstone, S., 1970. Nuclear reactor theory, Van Nostrand Reinhold Company.

Brockway, D., Soran, P., Whalen, P., 1985. LA-UR-85-1224.

Brun, E., et al., 2015. TRIPOLI- $4{ }^{\circledR}, C E A, E D F$ and AREVA reference Monte Carlo code, Ann. Nucl. Energy 82, 151-160.

Cao, Y., Lee, J.C., (2008). Nucl. Sci. Eng. 165, 270-282.

Cohen, E. R., 1958. In Proceedings of the 2nd UN conference of the peaceful uses of atomic energy, $\mathrm{P} / 629$, Geneva, Switzerland.
Duderstadt, J.J., Martin, W.R., 1979. Transport theory, J. Wiley and sons, New York.

Feghhi, S. A. H., Shahriari, M., Afarideh, H., 2007. Ann. Nucl. Energy 34, 514. Feghhi, S. A. H., Shahriari, M., Afarideh, H., 2008. Ann. Nucl. Energy 35, 1397.

Gandini, A., 1978. Nucl. Sci. Eng. 67, 91-106.

Gandini, A., 1981. Nucl. Sci. Eng. 79, 426-432.

Goad, W., Johnston, R., 1959. Nuc. Sci. Eng. 5, 371-375.

Henry, A. F., 1958. Nucl. Sci. Eng. 3, 52.

440 Henry, A.F., 1964. Nucl. Sci. Eng. 20, 338-351.

Henry, A.F., 1975. Nuclear Reactor Analysis, The MIT Press.

Hetrick, D.L., 1971. Dynamics of Nuclear Reactors, University of Chicago Press.

Hill, T.R., 1983. LA-9602-MS (UC-32).

Hoogenboom, J. E., 2003. Nuc. Sci. Eng. 143, 99.

Hurwitz Jr., H., 1964. In: Radkowsky, A. (Ed.), Naval Reactors Physics Handbook, vol. I. Naval Reactors, Division of Reactor Development, United States Atomic Energy Commission, pp. 864.

Keepin, G. R., 1965. Physics of Nuclear Kinetics, Addison-Wesley, Reading, UK.

Kiedrowski, B.C., 2010. LA-UR-10-01803.

Kiedrowski, B. C., 2011a. Adjoint Weighting for Continuous-Energy Monte Carlo Radiation Transport, $\mathrm{PhD}$ thesis, Michigan University.

Kiedrowski, B. C., Brown, F. B., Wilson, P. P. H., 2011b. Nucl. Sci. Eng. 68, 455

Larsen, E.W., Zweifel, P.F., 1974. J. Math. Phys. 15, 1987-1997.

Leppänen, J., et al., 2014. Ann. Nucl. Energy 65, 272.

Lewis, E.E., Miller, W.F.Jr., 1984. Computational Methods of Neutron Transport, J. Wiley and Sons.

Lux, I., Koblinger, L., 1991. Monte Carlo particle transport methods: Neutron and photon calculations, CRC Press, Boca Raton.

Mosteller, R. D., Kiedrowski, B. C., 2011. LA-UR-11-04409, Los Alamos National Laboratory.

Nauchi, Y., Kameyama, T., 2010. J. Nucl. Sci. Technol. 47, 977.

465 Nauchi, Y., 2014. In Proceedings of the SNA+MC 2013 conference, Paris, France.

Nolen, S.D., Adams, T.R., Sweezy, J.E., 2012. LA-UR-12-25458.

OECD Nuclear Energy Agency, 2010. International Handbook of Criticality Safety Benchmark Experiments. Report NEA/NSC/DOC(95)03.

Perfetti, C. M., 2012. Advanced Monte Carlo methods for eigenvalue sensitivity coefficient calculations, $\mathrm{PhD}$ thesis, Michigan University.

Persson, C.M., et al., 2008. Ann. Nucl. En. 35, 2357.

Pfeiffer, W., Brown, J.R., Marshall, A.C., (1974). GA-A13079, General Atomic.

475 Sanchez, R., Jaegers, P., 1998. LA-UR-98-336. 
Santamarina, A., et al., (2009). The JEFF-3.1.1 Nuclear Data Library, JEFF Report 22, OECD-NEA Data Bank.

dos Santos, A., et al. (2013) LEU-COMP-THERM-077: Critical loading configurations of the IPEN/MB-01 reactor, In International Handbook of Eval$480 \quad$ uated Criticality Safety Benchmark Experiments, NEA/NSC/DOC (95)03/I J. B. briggs Ed., Nuclear Energy Agency.

dos Santos, A., Diniz, R., 2014. Nucl. Sci. Eng. 178, 459.

Shim, H. J., Kim, C. H., Kim, Y., 2011. J. Nucl. Sci. Technol. 48, 1453.

Sjenitzer, B.L., Hoogenboom, J.E., 2013. Nucl. Sci. Engng. 175, 94-107.

485 Soodak, H., 1949. Pile kinetics. In: Goodman, G. (Ed.), The Science and Engineering of Nuclear Power, vol. II. Addison-Wesley Press, Inc., Cambridge, pp. 89102.

Truchet, G., et al., 2014. In Proceedings of the SNA+MC 2013 conference, Paris, France.

490 Truchet, G., et al., 2014. In Proceedings of the Physor 2014 conference, Kyoto, Japan.

Truchet, G., Leconte, P., Santamarina, A., Damian, F., Zoia, A., 2015. Ann. Nucl. Energy 81, 17-26.

Usachev, L. N., 1966. J. Nucl. Energy Part A/B 18, 571.

495 Ussachoff, L. N., 1955. In Proceedings of the 1st UN conference of the peaceful uses of atomic energy, $\mathrm{P} / 503$, Geneva, Switzerland.

Weinberg, A.M., 1952. Am. J. Phys. 20, 401-412.

Yamamoto, T., Miyoshi, Y., 2003. In Proceedings of the 7th Int. Conf. Nuc. Crit. Safety, ICNC 2003, Tokai-mura.

500 Ye, T., Chen, C., Sun, W., Zhang, B., Tian, D., 2006. In Proceedings of the 2006 Symposium on nuclear data, Tokai-mura, Japan.

Zoia, A., Brun, E., Malvagi, F., 2014. Ann. Nucl. Energy 63, 276.

Zoia, A., Brun, E., Damian, F., Malvagi, F., 2015. Ann. Nucl. Energy 75, 627.

Zoia A., Brun, E., 2016. Annals of Nuclear Energy 90, 71-82.

505 Zoia, A., Nauchi, Y., Brun, E., Jouanne, C., 2016. Annals of Nuclear Energy 96, 377-388. 